\title{
Run-Length Coding Algorithm Based Satellite Image Compression
}

\author{
Lakshmi $^{* 1}$, E.A. Mary Anita ${ }^{2}$ \\ ${ }^{1}$ AMET University, Chennai. \\ ${ }^{2}$ S.A. Engineering College, Chennai.
}

\begin{abstract}
Image compression is an application based on data compression of the digital images. Its main objective is to reduce the redundancy of the image data for storing and transmitting data in an easy way. In this system we are proposing a compression technique based on the Run-length coding algorithm based on satellite image compression. The Run-length coding algorithm is a part of the Lossless compression algorithm. The performance evolution can be done by calculating the PSNR values of the compressed images.
\end{abstract}

Keywords: data compression, run-length, lossless, PSNR.

\section{Introduction}

Imaging domains that deal with the use of image compression consists of the best existing compression method, with respect to image quality and are the lossless compression methods and is discussed here.

\section{Background}

A method to increase the compression ratio with less computational burden is discussed in [1]. In order to decomposition of a sub-block into equal sized bands, the DCT is used as a bandpass filter and a high similarity property is found among the bands.

The paper discussed in [2] uses the hybrid algorithm for the image compression technique. The hybrid algorithm is DWT for achieving a higher compression ratio and the DCT with the Huffman algorithm is used to preserve the quality of the reconstructed image. Region of interest (ROI) based on compression of image is discussed in [3] for classification applications. The method is a fusion of both lossy and lossless compression along with wavelets transforms like Embedded Zero-Tree Wavelet (EZW) is as derived techniques.

An adaptive sampling algorithm is used in [4], for evaluating the area difference between the predicted and the correct points to decide the significant coefficients. A linear equation is employed on the decoder side for the reconstruct of the coefficients between two sequent significant coefficients. An improved SPIRT algorithm in which most of the energy is intense in the low frequency sub-band after wavelet transform is discussed in [5]. By using the methods in wavelet tree construction and the diversity judgment rules of changing the original algorithm, the initial stage of the zero tree scan number is reduced. An image compression method based on Integer Wavelet Transform (IWT) and SVD is discussed in [6]. A graph based quantization is used in the method but the adaptive Huffman coding is used for entropy encoding. Also the IWT used here because it is reversible.

The discrete wavelet transform (DWT) [7] has gained wide popularity due to its excellent decorrelation property, many modern image and video compression systems embody the DWT as the transform stage [8]. It is widely recognized that the 9/7 filters are among the best filters for DWT-based image compression. After DWT was introduced, several codec algorithms were proposed to compress the transform coefficients as much as possible [9]. Among them, Embedded Zero tree Wavelet (EZW), Set Partitioning in Hierarchical Trees (SPIHT) and Embedded Bock Coding with Optimized Truncation (EBCOT) [10] are the most famous ones. An efficient video compression technique as modified HEVC coding based on saliency features to counter the existing drawbacks is introduced in [11]. Comparative Study of Wavelet Image Compression: JPEG2000 Standart using DWT and lead to an image data 
compression while preserving the essential quality of the original image is discussed in [12]. Discrete Wavelet Transform Based Image Compression using Frequency Band Suppression and Throughput Enhancement is described in [13]. The Security of Arithmetic Compression Based Text Steganography Method is explained in [14].

\section{The Problem}

The main drawback is that users cannot control the image quality over the background region or between regions with different degree of interest. For solving network bandwidth, security problems and storage problems, image need to be compressed.

\section{Proposed Solution}

The proposed methodology of our system is based on the satellite image compression algorithm known as run-length coding which is a type of the lossless based image compression. This method is implemented by using the extraction process of the ROI regions from the original satellite images and is compressed and decompressed by means of the run-length algorithm. The black diagram of the system is as shown in Figure 1.

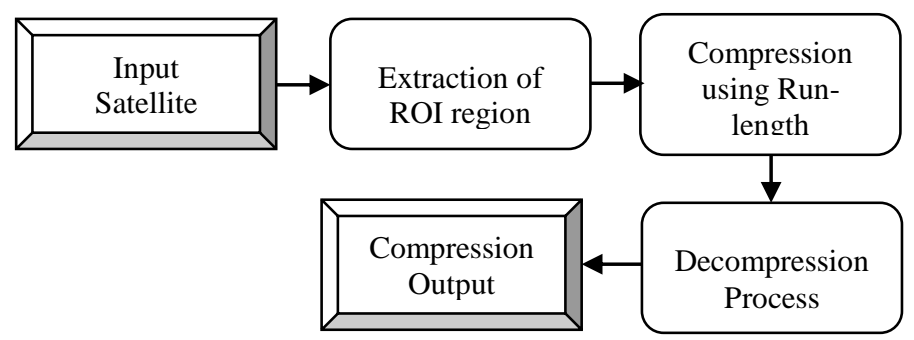

Figure 1. Block diagram for the proposed image compression Method

\subsection{Compression Process}

In the compression process first the $\mathrm{ROI}$ regions are extracted from the original satellite image by means of the FCM based segmentation process. The ROI images are extracted and then it is used for the compression process. The compression is done by means of the compression algorithm known as the run-length coding process. In this algorithm the neighboring pixels present in a typical image are correlated highly to each other. Also it is more economical to transmit the pixel value if there is a long run instead of encoding individual pixel values.

\subsection{Decompression Process}

After the extraction compression of the ROI images with the help of the run-length coding algorithm the compressed images are decompressed by revering the process of the runlength coding algorithm so that the image are and the data are retrieve back from the compressed image and the decompressed image is obtained by means of the decompression process. Here no data are lost during this process and so the image is compressed and decompressed by the run-length coding algorithm.

\section{Results and Discussion}

The run-length coding based image compression technique form the ROI extracted from the satellite images are based on the lossless compression process. In this process the images are compressed without any data loss from the image and so only the size of the image are reduced. The output result is as shown in Figure 2. 


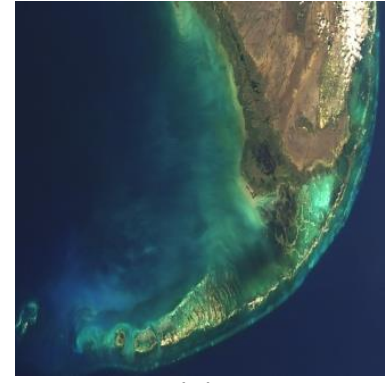

(a)

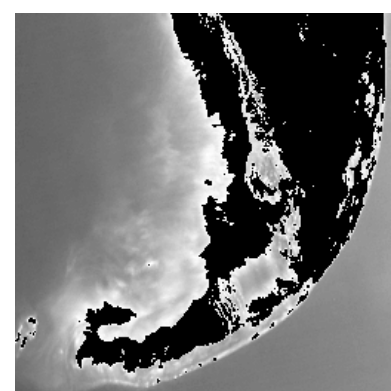

(c)

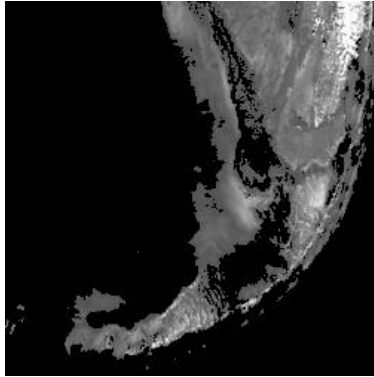

(b)

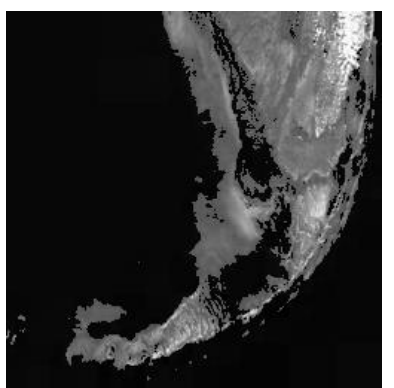

(d)

Figure 2. (a) Original image (b) Extracted ROI (c) Run-length compressed image

(d) Decompressed output image

\section{Conclusion}

The proposed image compression method based on the ROI extracted based lossless compression method by using the Run-length coding algorithm is discussed. The method is tested by the literally available satellite images. And the performance of the proposed system is analyzed by calculating the PSNR values of the compressed image. Our proposed system has obtained PSNR values of about $92.86 \% \mathrm{db}$.

\section{Reference}

[1] Wu YG, Tai SC. Medical image compression by discrete cosine transforms spectral similarity strategy. IEEE Transactions on Information Technology in Biomedicine. 2001; 5(3): 236-243.

[2] Sharma S, Bhat U. Image Compression using an efficient hybrid algorithm. 2013.

[3] Reddy BV, Reddy PB, Kumar PS, Reddy AS. Lossless Compression of Medical Images for Better Diagnosis. IEEE $6^{\text {th }}$ International Conference on Advanced Computing. 2016: 404-408.

[4] Wu YG. Medical image compression by sampling DCT coefficients. IEEE Transactions on Information Technology in Biomedicine. 2002; 6(1): 86-94.

[5] Bin L, Qinggang M. An improved SPIHT wavelet transform in the underwater acoustic image compression. IEEE International Conference on Measurement, Information and Control. 2013; 2: 1315-1318.

[6] Savitri PAI, Murdiansyah DT, Astuti W. Digital medical image compression algorithm using adaptive Huffman coding and graph based quantization based on IWT-SVD. IEEE $4^{\text {th }}$ International Conference on Information and Communication Technology. 2016; 1-5.

[7] Olivier Rioul, Martin Vetterli. Wavelets and Signal Processing. IEEE Trans. on Signal Processing. 1991; 8(4): 14-38.

[8] Panda SS, Jena G, Sahu S. Image super resolution reconstruction using iterative adaptive regularization method and genetic algorithm. Computational Intelligence in Data Mining. 2015; 2: 675681.

[9] Upadhyay A, Shetty A, Singh SK, Siddiqui Z. Land use and land cover classification of LISS-III satellite image using KNN and decision tree, IEEE 3rd International Conference on Computing for Sustainable Global Development (INDIACom). 2016: 1277-1280.

[10] Said A, Pearlman WA. A New Fast and Efficient Image Codec Based on Set Partitioning in Hierarchical Tree. IEEE Trans. on Circuits and Systems for Video Technology. 1996; 6(3): 243-250.

[11] Rekha B. High Definition Video Compression Using Saliency Features. Indonesian Journal of Electrical Engineering and Computer Science. 2017; 7(3). 
[12] Ennaciri A, Erritali M, Mabrouki M, Bengourram J. Comparative Study of Wavelet Image Compression: JPEG2000 Standart. Indonesian Journal of Electrical Engineering and Computer Science, 2015; 16(1): 83-90.

[13] Lakshmi Narayanan K, Ramesh G P. Discrete Wavelet Transform Based Image Compression using Frequency Band Suppression and Throughput Enhancement. International Journal of MC Square Scientific Research, 2017; 9(2): 176-182.

[14] Saniei R, Faez K. The Security of Arithmetic Compression Based Text Steganography Method. International Journal of Electrical and Computer Engineering, 2013; 3(6): 797. 